\title{
Haptic cooperation between people, and between people and machines
}

\author{
Kyle B. Reed and Michael Peshkin \\ Laboratory for Intelligent Mechanical Systems \\ Northwestern University \\ Evanston, IL, 60208 \\ \{reedkb, peshkin\}@northwestern.edu
}

\author{
Peter M. Vishton \\ Department of Psychology \\ College of William and Mary \\ Williamsburg, PA 23187 \\ vishton@wm.edu
}

\author{
Mitra J. Hartmann \\ Biomedical Engineering \\ Northwestern University \\ Evanston, IL, 60208 \\ m-hartmann@northwestern.edu
}

\author{
James Patton \\ Rehabilitation Institute of Chicago \\ Chicago, IL 60611 \\ j-patton@northwestern.edu
}

\author{
Marcia Grabowecky \\ Department of Psychology \\ Northwestern University \\ Evanston, IL 60208 \\ grabowecky@northwestern.edu
}

\begin{abstract}
Haptic interaction between people and machines might benefit from an understanding of haptic communication between one person and another. We recently reported results showing that two people performing a physically shared dyadic task can outperform either person alone, even when the perception of each participant is that the other is a hindrance [1]. Evidently a dyad quickly negotiates a more efficient motion strategy than is available to individuals. This negotiation must take place through a haptic channel of communication, and it is apparently at a level below the awareness of the participants. Here we report results on the motion strategy that emerged. By recording forces and motions we show that the dyads "specialized" temporally such that one member took on early parts of the motion and the other late parts. Tests in which one participant's contribution was surreptitiously replaced by a motor did not elicit a similar cooperative response from the remaining human participant, showing that the language of haptic communication between people must be rather subtle.
\end{abstract}

\section{INTRODUCTION}

Physical interaction occurs when two people exchange an object, jointly maneuver a bulky object, teach manual skills, or dance. Shared physical tasks require participants to adapt, anticipate, and react to each others forces and motions and are often performed in the absence of any explicit verbal communication. Forces and motions may be coupled directly limb-to-limb, or via a mutually grasped object.

We expect that an understanding of how two people physically cooperate, compromise, and guide one another may allow us to improve the cooperation and physical communication between a person and a machine. To make human-machine interactions intuitive to users, we expect it will be based upon natural human-human interactions, which are not yet well understood. Although there have been studies demonstrating robots working with humans [2][3][4], these controllers are not based upon the interactions of two people, they predominantly try to stabilize the system while the human manipulates the object.

\section{RElAtEd StUdiES}

Sallnas and Zhai [5] demonstrated the importance of force feedback for two people working together, showing that it significantly decreased the error rate for exchanging objects. They used a haptic system (rather than direct physical contact) to study performance during the handoff of a virtual object from one person to another. Whereas accuracy improved with feedback, the time to move the object was the same with and without force feedback, presumably because the interaction was brief.

In a study focusing on the forces between two people told to apply the same force as they felt, Shergill et al. [6] showed that two participants will escalate their force levels in repetitive physical contact. Participants in this experiment were told to apply the same force back to the other participant, yet participants continued to push back harder than they were pushed upon. This task involved only the communication of total force between the participants with no motions involved.

On motor tasks that require accuracy, people generally prefer to work alone, but past research has shown that two individuals can perform better than individuals on certain tasks. Wegner and Zeaman [7] reported that teams of 2 and 4 people were more accurate than an individual on a pursuit rotor. They were unable to suggest a satisfying explanation, possibly since they did not measure the forces exerted by the participants. Reed et al. [1] found that two people can perform a discrete target acquisition task faster than individuals acting alone. Gentry et al. [8] found a similar result for cyclical target acquisition tasks.

There has been surprisingly little research on physical cooperation between two people; we have found only the studies described above. In this paper, we examine the physical interaction between two people cooperating on a simple target acquisition task and investigate how to replace one person with a motor. This task revealed an emergent behavior where the dyad developed a new strategy for completing the task. 


\section{Method (Two-People)}

\section{A. Participants}

Thirty students (10 men; 2 left-handed), age 18-24, from Northwestern University's Psychology participant pool participated after giving informed consent.

\section{B. Stimuli and Apparatus}

Two randomly selected participants standing on opposite sides of the crank (Figure 1) held the rigidly connected and freely spinning handles. A bold black line displayed the handles angular position and a projector displayed the same target region for each participant. Participants were instructed to move the handle into the target region as quickly as possible and to hold it there until a new target appeared (at a randomly selected time between 700 and $1700 \mathrm{~ms}$ ). The target changed color when the handle was within the target region. Each target subtended $6^{\circ}$ of the $50.5 \mathrm{~cm}$ diameter disk $(2.6 \mathrm{~cm}$ at the perimeter of the disk) with a distance between consecutive targets of $70^{\circ} \pm 10^{\circ}(30.9 \pm 4.4 \mathrm{~cm})$. Five-sixths of the trials required a reversal of handle rotation from the previous trial; in one-sixth of the trials, handle motion in the same direction was required (catch-trials). We discarded catch trials and the trials immediately following a catch trial. The variation of the target position and the variation of the delay before a new target appeared were included to prevent subjects from adapting to a predictable pattern. The projector also displayed a performance measure to encourage participants to perform as well as possible.

\section{Procedure}

An experimental run started with an individual, or a dyad, performing a block of 120 trials (target acquisitions). Half of the participants completed one block of trials individually, and then one block as a dyad (A, B, AB). The other half performed as the dyad first ( $\mathrm{AB}, \mathrm{A}, \mathrm{B})$. The sequence was performed twice (e.g. A, B, AB, A, B, AB). Each block of 120 trials included 100 alternating-direction trials and 20 same-direction catch-trials. The experimental apparatus was identical when the participants were working as individuals and dyads, except that the rotational inertia of the crank $\left(0.113 \mathrm{~kg} \times \mathrm{m}^{2}\right)$ was doubled in the dyad condition. The entire experiment took less than 30 minutes, for a total of 720 non-discarded trials (480 for each participant). Force and common motion was recorded at a sampling interval of $1 \mathrm{msec}$.

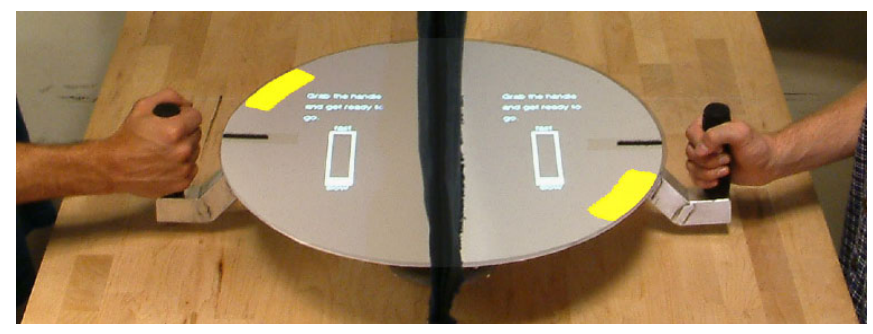

Fig. 1. Experimental setup with two-handled crank. A projector displays the targets from above. A curtain hangs between the two participants to prevent visual communication.

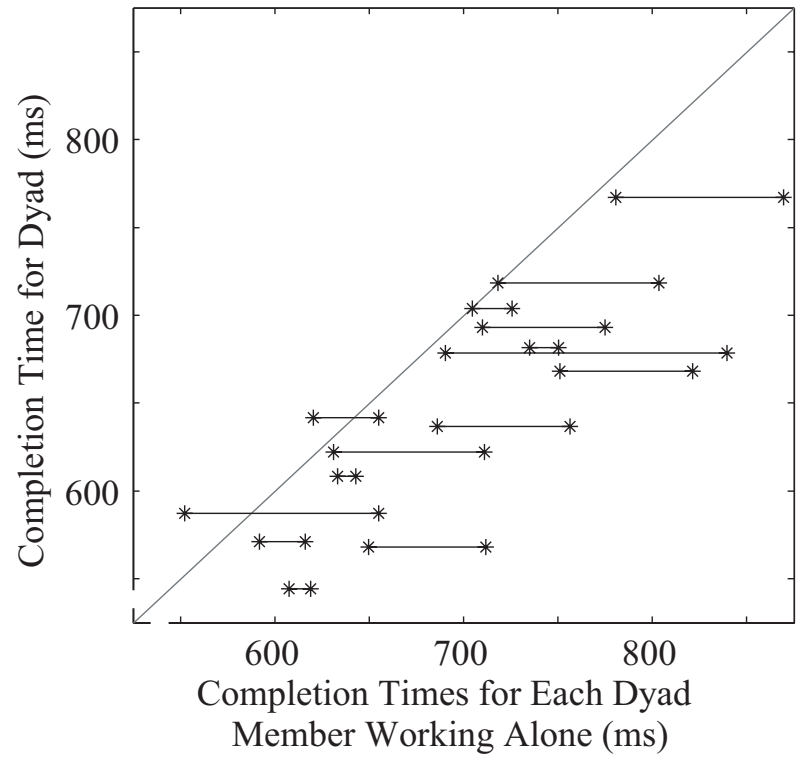

Fig. 2. This graph shows completion times for each individual performing alone (x-coordinates of the two "**" symbols) and for the same two individuals as part of a dyad (y-coordinate of the "**" symbols). A horizontal line connects the two members comprising each dyad. Hence, symbols to the left of the diagonal represent individuals who outperformed their dyad (only two of thirty). Dyads performed faster than individuals, despite expending significant force in opposition to each other. This figure was copied with permission from [1]

\section{PERFORMANCE}

We report results on the speed of task execution in Psychological Science [1], and will summarize only briefly here. We found that in almost all cases dyads completed the task faster than either one of the members could complete it when working individually (Figure 2). The improved performance developed quickly (within 20 trials) when the dyad began working together. Many participants reported a perception of interference from the other; few reported cooperation. The explanation cannot be sharing the load because we doubled the crank's rotational inertia in the dyad condition. Rather, the initial symmetry of the task was broken with the participants taking on different roles in the task.

In this report we focus on the way in which a task was shared asymmetrically by the participants. We find that dyads adopted similar cooperative strategies, although some dyads did so to greater extents than others. Having identified the cooperative strategy and the two roles that were commonly developing between people, we replaced one person with a robot which performed one of the roles, to see if the remaining person would adopt the other role.

\section{RESUlts AND Discussion (SPECIALIZATION)}

We measured the forces exerted by each member of each dyad in order to investigate this new completion strategy. The sum of the members forces ("net force") is the taskrelevant force that results in acceleration of the crank. The difference of their forces ("difference force") is a measure of the disagreement of the members, and has no physical effect 

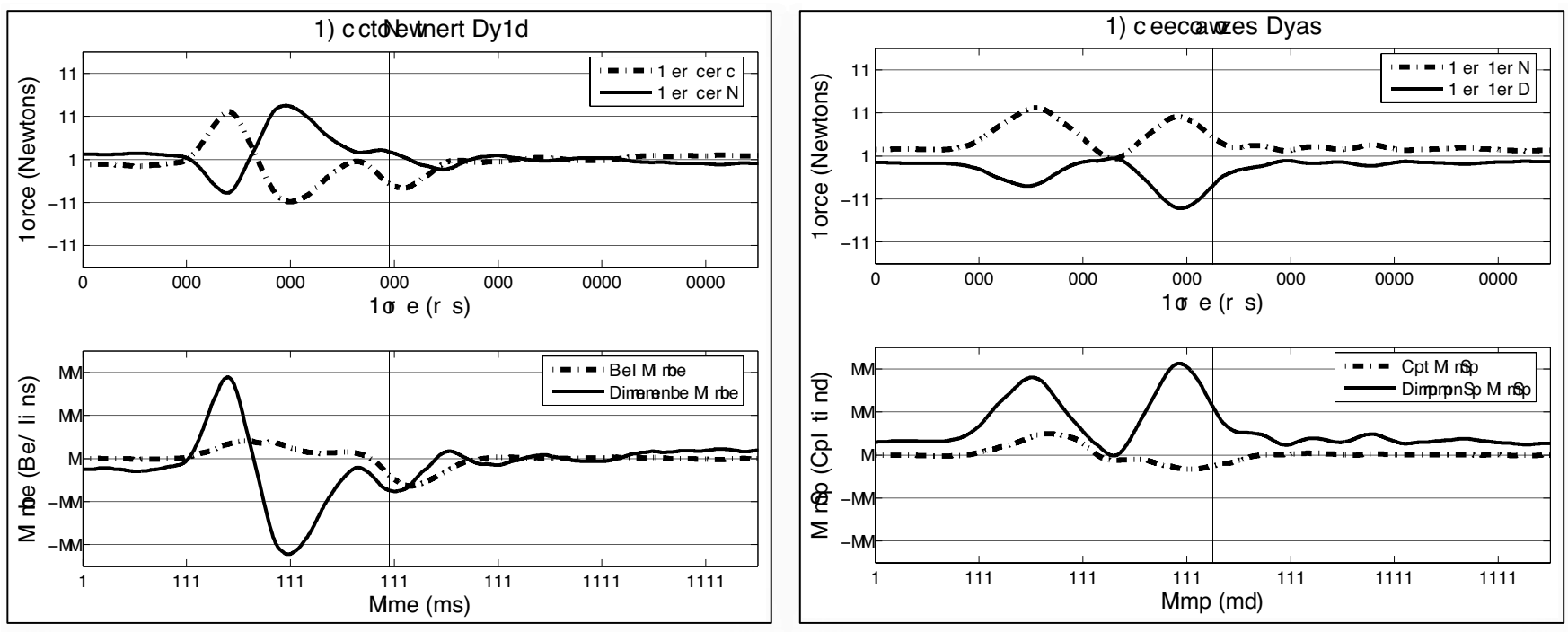

Fig. 3. Forces in a single trial for (a) an "active/inert" dyad and (b) a "specialized" dyad. The upper graphs show the forces produced by each member of the dyad when working together. Forces are recorded as positive when applied toward the target. The lower graphs show the sum and difference of their forces: the net force (the sum) contributes to crank motion while the difference force is expended in opposition to one another. In the specialized dyad, the difference force is always the same sign: member $\mathrm{C}$ accelerates the crank forward while $\mathrm{D}$ is pulled along, and then $\mathrm{C}$ continues to push forward (fails to reverse) while D brakes.

on crank motion. The difference force is thus a measure of the force expended that does not go into accelerating the crank; however, it is a possible channel of communication between the participants. Figure 3 shows the net, difference, and each member's force for a single trial by each of two dyads. The vertical line near $600 \mathrm{msec}$ in each plot indicates task completion (i.e., the time at which the cursor entered and stayed within the target).

An active/inert dyad is shown in Figure 3a. Early in the trial member A provides a force toward the target while participant B's passive inertia creates a counterproductive force. Late in the trial member A provides all the deceleration force as well, while member $B$ is again passive.

Figure $3 \mathrm{~b}$ shows a very different pattern, which we denote "specialized" - member C primarily contributes early in the trial (acceleration phase) while member D is pulled along. Member D primarily contributes late in the trial (deceleration phase) while member $\mathrm{C}$ continues pushing. This specialization is clearly revealed when inspecting the difference force, which remains always the same sign. Only the characteristic shape of the difference force matters - it could be mirrored around the $\mathrm{x}$-axis if the subjects were standing on the opposite sides.

To characterize the relative contributions of each member to the net motion of the crank, we developed a quantitative measure of specialization. We divided each trial into an acceleration phase and a deceleration phase, integrated the force contribution of each member over each phase, and divided by the integrated net force for that phase. The result provides the fractional contribution of each member of the dyad to the two phases (acceleration and deceleration). The two members' contributions necessarily sum to one. A negative contribution indicates that the member was working against the motion of the crank (accelerating during a deceleration phase, or decelerating during an acceleration phase, even if only due to passive inertia). A contribution greater than one indicates that this participant had to compensate for the negative contributions of the other dyad member.

Figure 4 shows two clusters of dots, one for a specialized dyad and one for an active/inert dyad, with each dot representing one trial. Each cluster includes only one member of a dyad, since data for the other member would be simply mirrored about the center of the box $(0.5,0.5)$. Dots near the $x=y$ line represent a member's equal contribution to acceleration and deceleration phases. The perpendicular distance of a dot from the $x=y$ line is thus a measure of the degree of specialization: a member contributing more to acceleration than to deceleration, or vice-versa.

To assess the significance of the observed acceleration/deceleration (A/D) specialization, we compared left/right (L/R) specialization - another reasonable division of the task, but one that was generally not adopted by our dyads. If dyads adopted L/R specialization, one member would contribute by pushing only to the left while the other member would contribute by pushing only to the right. Figure 5 compares A/D specialization to $\mathrm{L} / \mathrm{R}$ specialization. Eleven of the 15 dyads show significantly more A/D specialization than $\mathrm{L} / \mathrm{R}$ specialization $(p<0.05)$.

Knoblich and Jordon [9] suggest that when groups work together, they might perform better because they have fewer actions to deal with. In our dyad case, they specialize, which is one way they can get out of each others way and only deal with a few actions. Additionally, humans can precue an action so that when some other event happens they perform a certain action [10]. In the case of two people working together, the deceleration specialist waits for some event to 


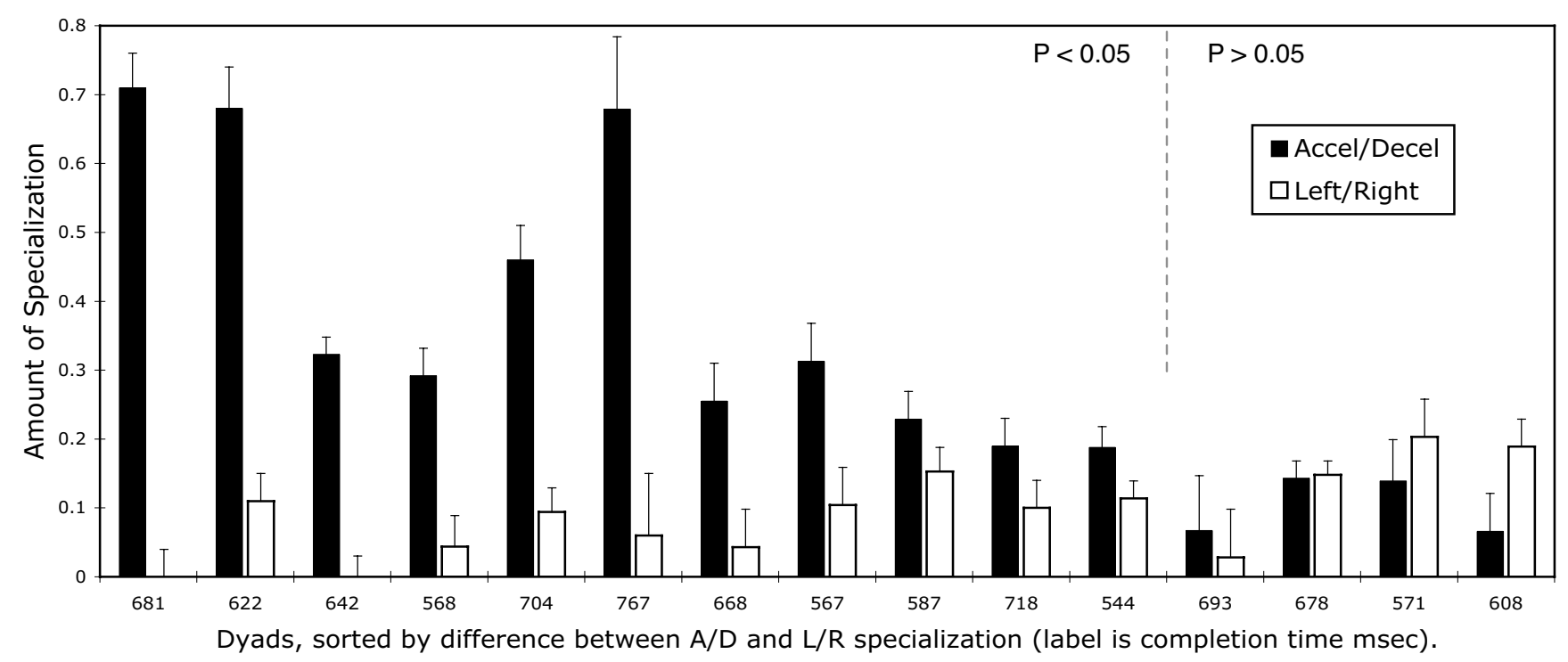

Fig. 5. Each bar shows a dyad's average degree of specialization over all trials, and a 95\% confidence interval. A value of 0.707 (the distance from the $x=y$ line to the point $(1,0)$, meaning one members contribution to acceleration is $100 \%$ and to deceleration is $0 \%$ ) can be considered "fully specialized." 11 of the 15 dyads show significantly more acceleration-deceleration (A/D) specialization (black bars) than left-right (L/R) specialization (white bars) $(p<0.05)$. Many, but not all, dyads show a qualitatively high degree of A/D specialization. The $\mathrm{x}$-axis label shows the average completion time for the given dyad.

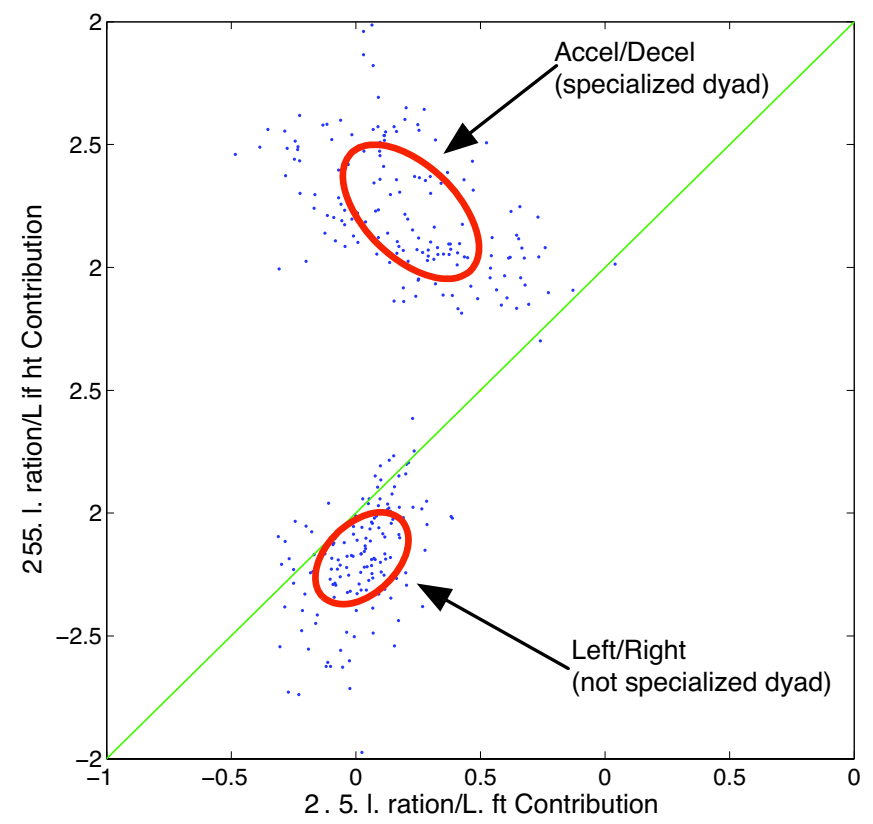

Fig. 4. Specialization by acceleration and deceleration phases of the movement. The graph shows superimposed datasets for two different dyads, one of which exhibits significant specialization. A dyads degree of specialization can be characterized by plotting one members contribution to the acceleration and deceleration phases. The other members contribution, not shown, is necessarily opposite, and would appear as a dot mirrored about the center of the box $(0.5,0.5)$. The distance from the $x=y$ line is the degree of specialization. The statistics of this specialization are captured in the ellipse. The center of the ellipse is the mean of the distribution, and the major and minor axes show one standard deviation. occur, such as reaching a certain location or velocity and subsequently begins to decelerate the crank.

A similar specialization strategy has been observed in a single individual performing a bimanual task [11]. Reinkensmeyer et al. show that an individual holding a pencil between two fingers on different hands will accelerate with one hand and decelerate with the other hand, which might be taken as a bimanual model for our observed two person A/D specialization. The tight neural coupling between two arms in an individual allow very different strategies to be used [12]. For a single individual the acceleration and deceleration allows the pencil to move while being rigidly held and not dropped. In our dyadic task there is, of course, no neural coupling between the individuals. The developed strategy must have occurred by haptic communication instead since the only communication between participants was physical, whereas in the bimanual pencil task the communication was internal to one person. Since two people can perform a strategy similar to a single person, would a simulated arm that plays back half of a recorded trajectory be enough to elicit the same response? The next experiment examines this question.

\section{Method (Person-Motor)}

\section{A. Participants}

11 students (3 men; 1 left-handed), age 18-24, from Northwestern University's Psychology participant pool participated after giving informed consent.

\section{B. Stimuli and Apparatus}

The same experimental apparatus shown in Figure 1 was used, but with one person and a simulated person using a motor mounted under the table. The motor simulated a 
second person by playing back an average trajectory of the subjects' own force during individual trials. The force was only displayed during the acceleration phase to elicit specialization. The force was multiplied by 2.1 , which is the amount an individual increased his force by when he becomes part of a dyad [1]. Applying this motor force would allow them to become a decelerator while the motor becomes the accelerator, thus specializing with a simulated arm.

In order to simulate the robotic arm, an inertia equal to a human arm was added. The inertia of a human arm was measured by grabbing the crank with the same grip used in the experiments and applying a torque from the motor and measuring the acceleration over a frequency range from 1 to $35 \mathrm{~Hz}$. To account for accelerating that extra inertia, the robotic force during the acceleration phase force was also increased proportionately.

A confederate, who pretended to be an actual subject, arrived and stood on the opposite side of the crank to make each participant believe that they were working with a real person (i.e not a motor). 10 of the 11 participants said they thought they were working with a person. One of them had some doubts, but was not sure either way and the results were not significantly different.

\section{Procedure}

An experimental run started with an individual (I) or the confederate (C) performing a block of trials individually. The confederate appeared unknowledgeable of the experiment and performed individual trials to make it appear that they were participants. The procedure for this experiment was the same as the above experiment, except the dyad condition $(\mathrm{AB})$ is now a participant working with the motor simulating a person $\left(\mathrm{I}_{m}\right)$. The sequence was $\left(\mathrm{I}, \mathrm{C}, \mathrm{I}_{m}, \mathrm{I}, \mathrm{C}, \mathrm{I}_{m}\right)$ for half the participants and $\left(\mathrm{C}, \mathrm{I}, \mathrm{I}_{m}, \mathrm{C}, \mathrm{I}, \mathrm{I}_{m}\right)$ for the other half. There were no catch trials since the learned trajectory only accounted for the standard back and forth trials and a lack of motor force would add clues that the confederate was not actually participating.

\section{RESUlts AND Discussion (COMPARISON)}

The Two-Person experiment had catch trials while the Person-Motor experiment did not, but the difference in completion times (CT) between one person and a dyad (or person and motor) is comparable between experiments. Any difference of having or not having catch trials should be eliminated because we are looking at the change in completion time within each experiment.

When two people work together, the dyad performs on average $54.5 \mathrm{~ms}$ faster than they do alone. When a person works with the above force trajectory, they are on average $5.8 \mathrm{~ms}$ faster than they are when working alone. There is a significant difference of $48.8 \mathrm{~ms}$ between the Two-Person and Subject-Motor completion times $(t(11,15)=3.02, p=$ 0.006).

With a subjects' own amplified force applied as a feedforward force, the subjects are given an easy way to specialize. There is no motor applied force during deceleration, so the

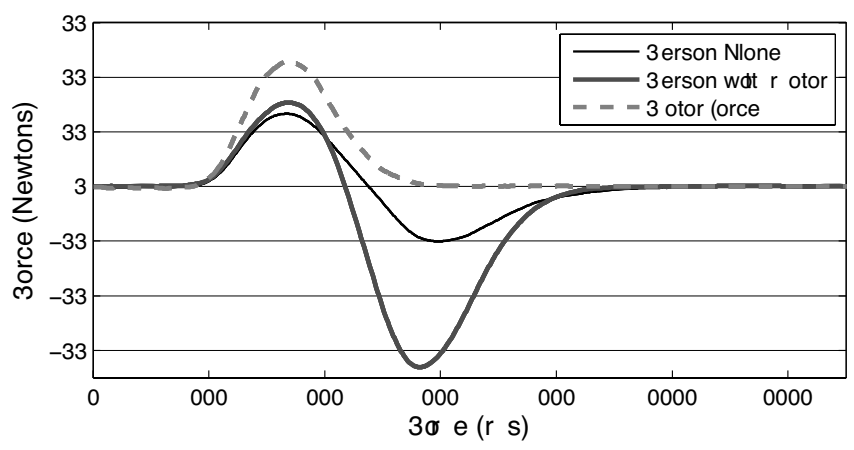

Fig. 6. Forces averaged over all subjects for both the trials when working alone (light) and the trials when working with the simulated person (dark). The forces are very similar in both cases. The acceleration phase (positive) changes only slightly when the subjects work with a motor that provides enough force to accelerate the handle toward the target. The deceleration phase (negative) is very different because there is no motor force and the subject is required to apply all the force to stop the crank. This shows that a simple force trajectory is not sufficient to elicit specialization in a person. There are more forms of haptic communication that are required.

subject is required to apply all the force, whereas they are free to choose their force during the acceleration phase. Comparing the applied force from a person working alone to a person working with a motor shows that they do not change their feedforward torque much during the acceleration phase. Figure 6 shows the average motion of all subjects alone compared to the same subjects working with the motor. They tend to accelerate similarly and actually apply slightly more peak force, but switch to deceleration earlier than they do when working alone. It seems that the subjects are pushing with a preprogrammed feedforward torque similar to their previous trials alone, but begin to correct it and slow down the crank earlier. For a brief period, they are actually pushing against the motor. Compared to two specialized people working together (Figure 3b), this force profile shows a very different strategy that is very similar to an individual performing the task. None of the subjects developed specialization like two people working together did.

When working as a dyad, each member does not know exactly what the result of their action will be since it will be combined with another members' action. Sebanz et al. [13] show that a person will internally represent the actions of a person nearby when working on a complementary action. Working with a partner haptically would presumably develop a similar internal representation, which would allow each subject in a pair to depend upon the other member, thus allowing them to specialize to different aspects of the task. Simply playing back a recorded force possibly does not elicit such a representation to develop since this force is solely feedforward and it does not adapt to the subject.

Since the subjects are only adapting slightly (and not specializing) and their performance gets worse, it is apparent that this playback approach does not have the benefits of working with another human. This approach is missing an aspect of how two people work together, presumably some sort of feedback, adaptation throughout trials, and other types of haptic communication. 


\section{FUTURE WORK}

This simple feedforward trajectory does not elicit a specialized role to develop, despite encouraging the subjects to do so. When working with another human, there are quite possibly cues that develop to indicate certain transition points, such as when to transfer control or when to start pushing the other direction. In future experiments with two people working together, we could remove certain aspects of physical communication between the subjects, such as notch filtering all $8 \mathrm{~Hz}$ noise (which is a common frequency that the human nervous system operates at) or high/low pass filtering the forces that are displayed to each person. If the performance decreases, then this aspect of communication is presumably important and would be added back into a new version of the simulated person.

\section{ACKNOWLEDGMENTS}

The authors wish to thank J. Edward Colgate, Kevin Lynch, and Satoru Suzuki for their help with this research. This work was supported by NSF grant ECS-0433948.

\section{REFERENCES}

[1] Kyle Reed, Michael Peshkin, Mitra J. Hartmann, Marcia Grabowecky, James Patton, and Peter M. Vishton. Haptically linked dyads: are two motor control systems better than one? Psychological Science, 17(5):365-366, 2006.

[2] Weston B. Griffin, William R. Provancher, and Mark R. Cutosky. Feedback strategies for telemanipulation with shared control of object handling forces. Presence, 14(6):720-731, 2005.
[3] Paul Griffiths and R. Brent Gillespie. Shared control between human and machine: Haptic display of automation during manual control of vehicle heading. Haptics 2004, pages 358-366, 2004.

[4] T. Takubo, H. Arai, Y. Hayashibara, and K. Tanie. Human-robot cooperative manipulation using a virtual nonholonomic constraint. The International Journal of Robotics Research, 21:541-553, 2002.

[5] E. Sallnas and S. Zhai. Collaboration meets Fitts' law: Passing virtual objects with and without haptic force feedback. Proceedings of INTERACT, IFIP Conference on Human-Computer Interaction, pages 97-104, 2003.

[6] S. S. Shergill, P. M. Bays, C. D. Frith, and D. M. Wolpert. Two eyes for an eye: The neuroscience of force escalation. Science Magazine, 301:187, 2003.

[7] N. Wegner and D. Zeaman. Team and individual performance on a motor learning task. Journal of General Psychology, 55:127-142, 1956.

[8] Sommer Gentry, Eric Feron, and Roderick Murray-Smith. Humanhuman haptic collaboration in cyclical fitts' tasks. International Conference on Intelligent Robots and Systems, 2005.

[9] Gunther Knoblich and Jerome Scott Jordon. Action coordination in groups and individuals: Learning anticipatory control. Journal of Experimental Psychology: Learning, Memory, and Cognition, 29(5):1006-1016, 2003.

[10] D. A. Rosenbaum. Human movement initiation: Specification of arm, direction, and extent. Journal of Experimental Psychology: General, 109:444-474, 1980.

[11] David J. Reinkensmeyer, Peter S. Lum, and Steven L. Lehman. Human control of a simple two-hand grasp. Bilogical Cybernetics, 67(6):553564, 1992.

[12] Stephan P. Swinnen and Nicole Wenderoth. Two hands, one brain: cognitive neuroscience of bimanual skill. TRENDS in Cognitive Sciences, 8(1):18-25, 2004.

[13] Natalie Sebanz, Gunther Knoblich, and Wolfgang Prinz. Representing others' actions: just like one's own? Cognition, 88:11-21, 2003. 